\title{
Los pasatiempos como recurso didáctico en el aula de E/LE (2): herramientas en línea para la creación de crucigramas
}

\author{
MANUEL JosÉ AguiLAR RUIZ \\ Universitat de València \\ Manuel.J.Aguilar@uv.es
}

\begin{abstract}
Resumen: El objetivo del presente artículo es ofrecer al docente de E/LE un análisis de tres herramientas (Crucigramas para imprimir, Educima y EclipseCrossword) para generar los pasatiempos que conocemos con el nombre de crucigramas. Nos centraremos en las variables básicas que este tipo de juego lingüístico ofrece para su configuración, como la elección del número de palabras, de las piezas léxicas en sí, de un campo léxico determinado, del nivel de dificulta o del tamaño de este. El artículo se integra en una serie de análisis y aprovechamiento de diversos pasatiempos en el aula de E/LE, iniciada en trabajos anteriores.
\end{abstract}

Palabras clave: Español, E/LE, pasatiempos, crucigramas.

\section{Puzzles as a didactic resource in the class of Spanish language (2): Online tools for creating crosswords}

Abstract: The aim of this paper is to offer the teacher of Spanish as a foreign language an analysis of three tools to generate the linguistic puzzles we know with the name of crosswords -"Crucigramas para imprimir", "Educima" and "EclipseCrossword". We will focus on the basic variables that this kind of game offer for its configuration, such as the choice of the number of words, the words themselves, a specific lexical field, the level of difficulty or $f$ the size of this puzzle. The paper joins a series of analysis and use of various puzzles in the classroom of Spanish as a foreign language initiated in previous papers.

Keywords: Spanish, Spanish as a Foreign Language, word puzzles, crosswords.

\section{Introducción}

En el presente artículo pretendemos continuar las observaciones sobre las altas posibilidades didácticas del empleo de pasatiempos de naturaleza lingüística en el aula de E/LE, iniciadas ya en un trabajo anterior (focalizado en los mensajes cifrados o criptogramas, en Aguilar Ruiz, 2019) y reconocidas por el Marco común europeo de referencia para las lenguas (MCER, 2002: 59). Aquí nos centraremos en el juego lingüístico que conocemos como crucigrama, esto es, el pasatiempo consistente en una parrilla compuesta por series horizontales y verticales que han de rellenarse con letras, de forma que han de formar las palabras que corresponden a unas definiciones dadas. Con esta publicación pretendemos, más que dotar al docente de E/LE de una batería de crucigramas ya realizados y que se encuentran rápida y fácilmente en la web o como 
material publicado ${ }^{1}$, ofrecer algunas herramientas útiles en línea para que este sea capaz de diseñar, personalizar y adecuar este tipo de juego a las necesidades del alumnado. Un resumen sobre los beneficios, capacidades didácticas y planteamientos metodológicos de los pasatiempos en general y de los crucigramas en particular puede encontrarse en Aguilar Ruiz (2019: 7-10).

\section{Herramientas en línea para la creación de crucigramas}

De la amplia oferta de generadores de crucigramas en español que ofrece la web, para nuestro análisis hemos elegido los tres que hemos venido empleando con más frecuencia en nuestra práctica docente: Crucigramas para imprimir, Educima y EclipseCrossword. Las dos primeras herramientas, analizadas en los apartados 2.1. y 2.2., son páginas webs con las que podemos crear en línea nuestros juegos y personalizarlos, mientras que la tercera no es propiamente un generador en línea, sino un programa instalable, y se examinará en 2.3 .

Nuestra selección se debe a que estos son, hasta donde tenemos noticia, los generadores que nos permiten mayor libertad a la hora de diseñar y personalizar este tipo de juego en concreto para adaptarlo a las necesidades específicas de los alumnos y poder trabajar, así, con ellos, un campo léxico determinado.

De esta manera, y frente a los pasatiempos que libros de texto o páginas web nos ofrecen ya creados, los generadores de crucigramas antes citados nos permiten controlar, en mayor o menor medida, variables básicas con las que podemos diseñar el juego, como son un campo semántico determinado, el nivel de dificultad, el número de unidades léxicas que se han de descubrir, la cantidad máxima de líneas horizontales y verticales, etc. Todos ofrecen la posibilidad de imprimir el resultado, con lo que podemos entregar el material impreso a los alumnos para que realicen las actividades propuestas de manera individual o en distintas agrupaciones o parejas, aunque, contando con un proyector en el aula, también podemos plantearnos la posibilidad de proyectarlo para su resolución en común. Dos de ellos, además, cuentan con la interesante característica de que son interactivos, de manera que el resultado puede resolverse en línea y desde el ordenador. Obviamente, la forma de resolución de la actividad será siempre de libre elección por parte del docente.

\subsection{Creación de crucigramas mediante la web Crucigramas para imprimir}

La página web Crucigramas para imprimir $^{2}$ nos ofrece la posibilidad de crear crucigramas eligiendo variables importantes de las que hemos hecho mención en el apartado anterior: un campo semántico concreto, el nivel de dificultad, el número de

\footnotetext{
1 Por ejemplo, en Lefebvre $\left(\begin{array}{lll}s . & d .\end{array}\right)$ en la web Todoele.net (http://www.todoele.net/actividades/Actividad_maint.asp?Actividad_id=368 (fecha de consulta: 10/03/2020), los Pasatiempos de Rayuela del Instituto Cervantes (en https://cvc.cervantes.es/aula/pasatiempos/pasatiempos1/) (fecha de consulta: 10/03/2020), ePasatiempos (en www.epasatiempos.es) (fecha de consulta: 10/03/2020), Olenych (2001), Jacquet y Casulleras (2006), Cohen y Frates (2010); etc.

${ }^{2}$ En http://www.crucigrama.org/index.php (fecha de consulta: 10/03/2020). Esta página web se llamaba anteriormente «Palabras que. El laboratorio de palabras», y se encontraba alojada en www.palabrasque.com/crucigramas/crucigramas.php.
} 
unidades léxicas, la cantidad máxima de líneas horizontales y verticales, etc., aunque no contamos con la posibilidad de controlar totalmente el diseño definitivo del juego, como se verá.

En primer lugar, situados en su página web, elegimos uno de los catorce campos semánticos que este generador en línea ofrece (animales, colores, palabras usuales, etc.), clasificados por niveles (1-fácil, 2-medio y 3-difícil) y con un máximo de palabras disponibles (por ejemplo, los nombres de los colores, de nivel 1-fácil, tiene un máximo de 17 palabras, mientras que el léxico de los animales, de nivel 2-medio, dispone de 163 lexías, etc.). Tenemos la posibilidad de seleccionar el número de palabras que entrarán en juego (aunque no las piezas léxicas en sí), además del número máximo de filas y columnas, el tamaño de la letra y de las casillas (mediante la marcación de la casilla «Tamaño grande»), etc. (vid. fig. 1).

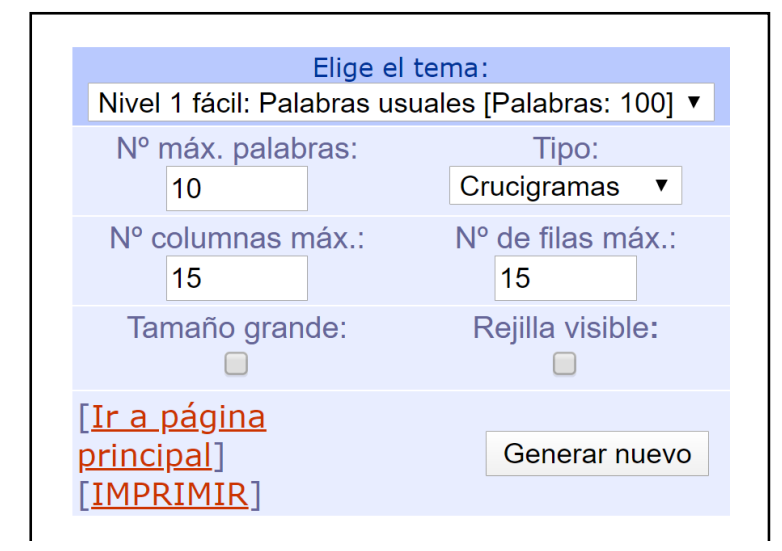

Figura 1. Cuadro inicial de configuración de la web Crucigramas para imprimir, en http://www.crucigrama.org/index.php.

El crucigrama se genera en unos segundos, y podemos originar, así, una gran variedad de crucigramas de manera rápida y sencilla. Véase, como ejemplo, la figura 2, compuesta por tres capturas de pantalla del resultado originado por esta web para un crucigrama centrado en el campo léxico de los colores, pensado para niveles como A2 o B1. 


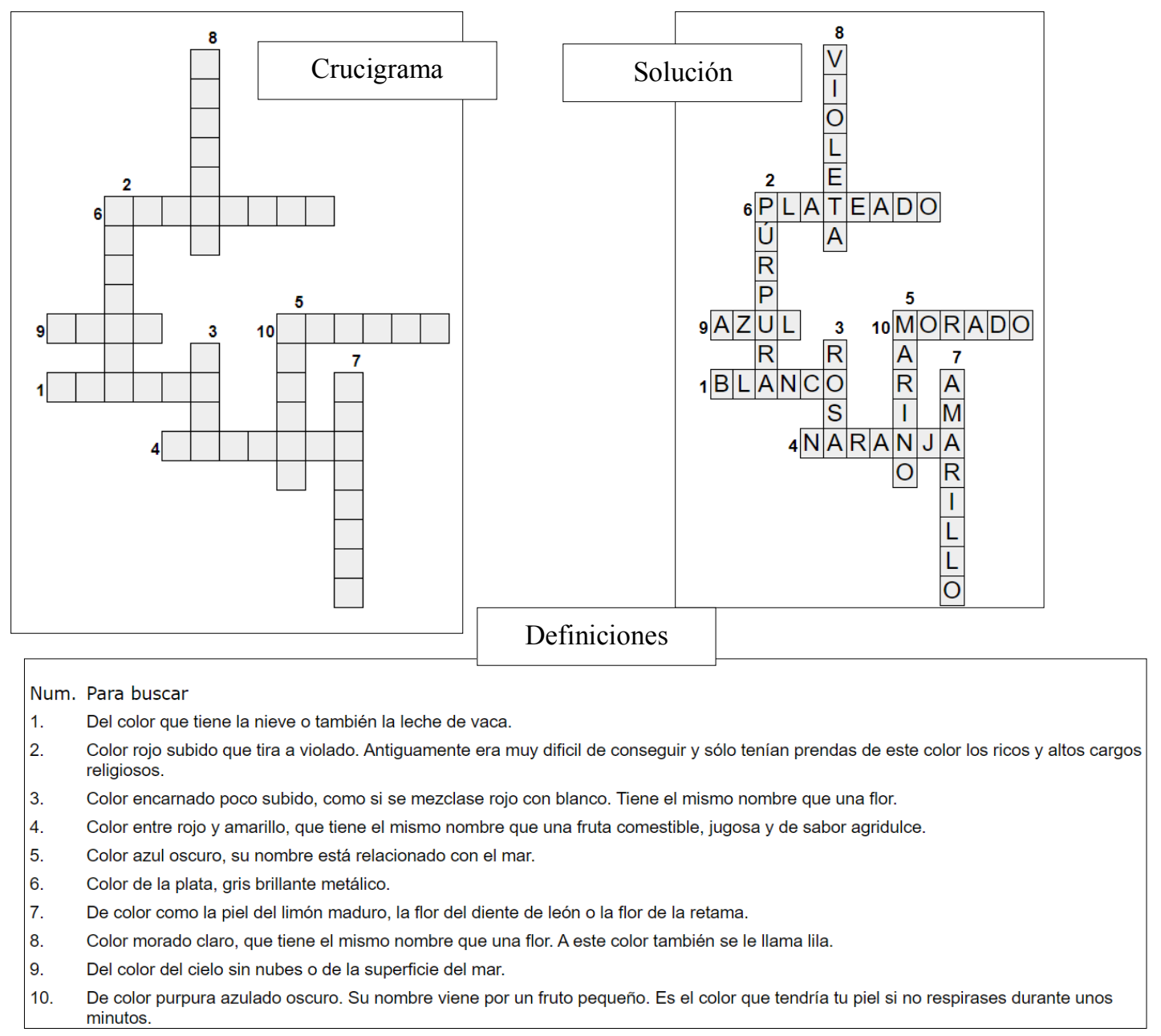

Figura 2. Crucigrama sobre el campo léxico de los colores, originado con la web Crucigramas para imprimir.

No obstante, como hemos adelantado, en esta web no contamos con la posibilidad de establecer las unidades léxicas específicas que precisemos, sino que las elegimos (estableciendo el número) de entre un inventario cerrado, aunque en ocasiones verdaderamente amplio. En efecto, algunos campos léxicos parecen sobrerrepresentados, como el de los animales: contamos con un máximo de 163 lexías sobre dicho campo, pero luego este aparece desdoblado en otros campos como «aves» (con 18 voces), «granja» (con 17), «insectos» (con 15), «lagartos» (con 9), «mamíferos» (con 39) y «marinos» (con 9). Otros campos aparecen ampliamente sobredimensionados cuantitativamente, de modo que mientras que temas como «palabras usuales»" ${ }^{3}$, «mitología» ${ }^{4}$, «psicología» ${ }^{5}$ o lo que esta web denomina «palabras eruditas» ${ }^{6}$ contienen un rango entre el centenar y las quinientas voces, el nivel 2-medio de léxico general

\footnotetext{
${ }^{3}$ Entre las que se encuentran aire, día, habitación, hermano, hija, idea, mar, pueblo, rey, rostro o vino.

${ }^{4}$ Con Anquises, Atenea, Ceres, Deucalión, Diomedes, Escila, Hidra, Hipólita, Ixión, Mnemosine, Minotauro, Mirra, Moira, Nereida, Pan, Pluto, Portunus, Psique, Ulises, Remo o Vertumno, entre otras.

${ }_{5}^{5}$ Entre las que se encuentran angustia, anorexia, astenia, autista, biotipo, catatonia, catecolamina, cognitivo, culpa, estereotipo, hábito, hedonismo, inconsciente, intersexual, onírico o social.

6 Con angosto, candor, enseres, escatológico, gallardo, heurística, insolente, ímpetu, implícito, misántropo, postrimería, pujo, recato, rocambolesco, sedicioso, subyacente, timidez, o univocar, entre otras.
} 
(denominado «definiciones») cuenta con más de veintiuna mil piezas léxicas ${ }^{7}$. El hecho de que el profesor no pueda seleccionar las voces concretas supone una circunstancia algo desfavorable; sin embargo, esta web sigue siendo una herramienta útil si se desea conseguir un crucigrama de forma sencilla y rápida, si en ese momento no nos importa tener el control total de las unidades léxicas que vayan a aparecer.

Un segundo inconveniente que encontramos en este generador de crucigramas en línea es que no contempla la posibilidad de incluir unidades léxicas morfológicamente superiores a la palabra, de modo que no podemos incluir colocaciones, compuestos sintagmáticos, locuciones u otras unidades léxicas pluriverbales de diverso tipo fraseológico ${ }^{8}$, tales como cajero automático, dibujos animados, tercera edad, tarjeta de crédito, hacer punto, dar plantón, un poco, a menudo, etc., que manuales y libros de texto suelen incluir ya desde los niveles iniciales (vid. Aguilar Ruiz, 2011: 2) y cuya docencia en el mundo E/LE suele contemplarse recurrentemente (Aguilar Ruiz, 2013; Gómez González y Ureña Tormo, 2014; Merino González, 2015; Puertas Ribés, 2019; etc.).

Otro gran problema que presenta esta página son las definiciones. Muchas están tomadas del diccionario académico, lo que puede ser un acierto si empleáramos este recurso en la docencia del español fuera de marco de una L2-LE, como en alumnos de español como L1, por ejemplo, pero esto ocasiona que muchas de estas definiciones puedan presentar cierta dificultad de inteligibilidad por parte de alumnos no nativos (e incluso nativos, en ocasiones), por su lenguaje anquilosado, definiciones interdependientes, «círculos viciosos», «pistas perdidas» y demás irregularidades y defectos que suelen achacársele a las definiciones del diccionario académico (vid. Medina Guerra, 2003: 144-146; Porto Dapena, 2014: 265-295). Para paliar este problema, el profesor podría obtener las definiciones de diccionarios especializados en la enseñanza de español, como el Gran diccionario de la lengua española (GDLE, 1985), el Diccionario para la enseñanza de la lengua española (DPELE, 1995), el Diccionario Salamanca de la lengua española (DSLE, 1996) o el Diccionario de español para extranjeros (DEPE, 2002), entre otros. Podrían también emplearse diccionarios de aprendizaje en general, como el Diccionario de uso del español actual (Clave, 1996) o el Diccionario estudio Salamanca (DESAL, 2007). Hay a disposición, además, algunos manuales y diccionarios especializados en la realización y resolución de crucigramas, como el Diccionario de crucigramas (1974), el Diccionario del crucigramista (1987), el Diccionario auxiliar del crucigramista (1995) o Saber resolver crucigramas (2003).

\subsection{Creación de crucigramas mediante la web Educima}

La página web Educima ${ }^{9}$, por su parte, contiene un generador de crucigramas que es una de las herramientas que hemos venido empleando para generar este tipo de pasatiempos

\footnotetext{
7 Entre otras, ambición, apatía, bestialidad, carmín, cinc, claxon, consejero, desfachatez, economía, elipsis, marojo, monstruoso, noguera, ocupar, pigmento, pata, pudrir, ronzal, significar, sordo o ventisca.

${ }^{8}$ Aunque habría que destacar que no es una práctica común ofrecer en crucigramas (o en sopas de letras) unidades léxicas pluriverbales (como compuestos sintagmáticos, colocaciones o locuciones), podríamos resolver de manera sencilla esta carencia si no contamos con los espacios en blanco entre las lexías que compongan una unidad léxica compleja.

${ }^{9}$ En https://www.educima.com/crosswordgenerator.php (fecha de consulta: 10/03/2020).
} 
y llevarlos al aula de español. Ofrece un número limitado de ítems para buscar (en concreto, 16), pero, a diferencia de la web anterior (Crucigramas para imprimir), tenemos total libertad para elegir las unidades léxicas específicas que deseemos (esto es, no vienen determinadas por la propia web); además es el docente el redactor de las definiciones, para las que se permite, asimismo, un gran número de caracteres (vid. figura 3). Divide las pistas en horizontales y verticales (por su modo de lectura en el juego) y ofrece la opción de variar el posicionamiento de las casillas y mostrar la solución. Hemos de recordar que no aporta las definiciones, por lo que las debe integrar el docente (extrayéndolas, por ejemplo, de alguno de los diccionarios especializados señalados antes, al final del punto 2.1.). Además, el crucigrama originado es interactivo, de modo que se puede solucionar desde la misma web, aunque ofrece también la posibilidad de descargarlo (como archivo de imagen) o imprimirlo.

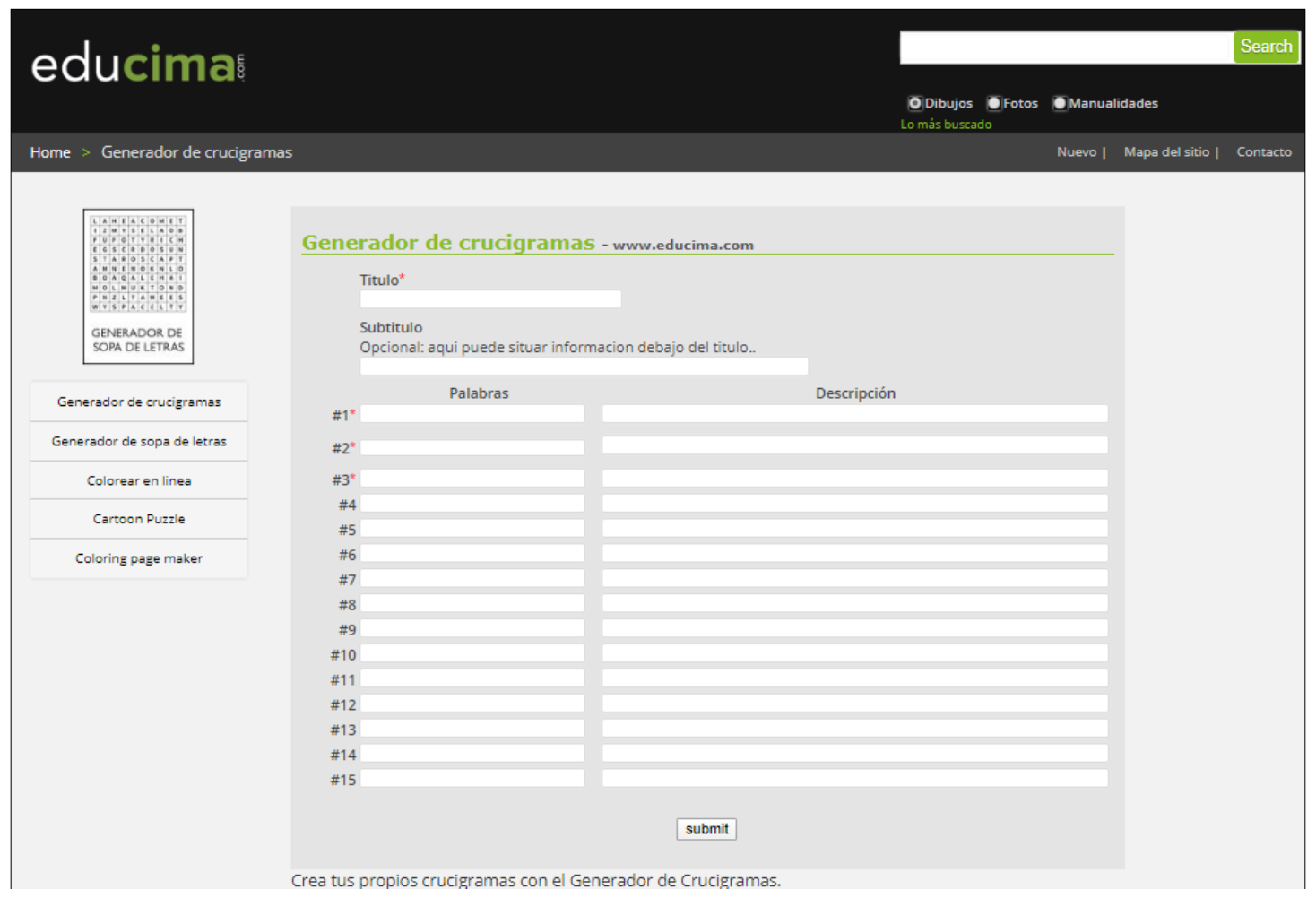

Figura 3. Captura de pantalla del generador de crucigramas de la web Educima (En https://www.educima.com/crosswordgenerator.php).

Como ejemplo, puede consultarse a continuación la figura 4, con un crucigrama realizado sobre el campo léxico de los animales ( $\mathrm{y}$, en concreto, sobre nombres de animales contenidos en locuciones usuales españolas). Con él se pretende reforzar el conocimiento de locuciones coloquiales que contienen el nombre de un animal como componente léxico, estudiadas previamente en el aula (por ejemplo, llevarse el gato al agua $^{10}$, no ver tres en un burro ${ }^{11}$ o tener malas pulgas $\left.{ }^{12}\right)$. En lugar de ofrecer una definición, ofrecemos como «pista» las locuciones concretas, cada una con una casilla vacía, la cual es la pieza léxica que se ha de anotar en el crucigrama (en los ejemplos

\footnotetext{
10 'loc. verb. col. En un enfrentamiento, triunfar o salir victorioso: La tenista estuvo a punto de perder, pero al final se llevó el gato al agua' (Clave, s. v. gato).

11 'loc. verb. col. Ver mal o ser miope: Con esta niebla, no veo tres en un burro' (Clave, s. v. burro).

12 'loc. verb. col. Tener mal genio o enfadarse con facilidad' (Clave, s. v. pulga).
} 
anteriores, llevarse el al agua, no ver tres en un $\mathrm{y}$ tener malas respectivamente), como suelen ofrecerse en ocasiones este tipo de pistas (vid. Gómez Molina, 2000; Ruiz Gurillo, 2000; Aguilar Ruiz, 2012, 2013; Gómez González y Ureña Tormo, 2014 y 2017).

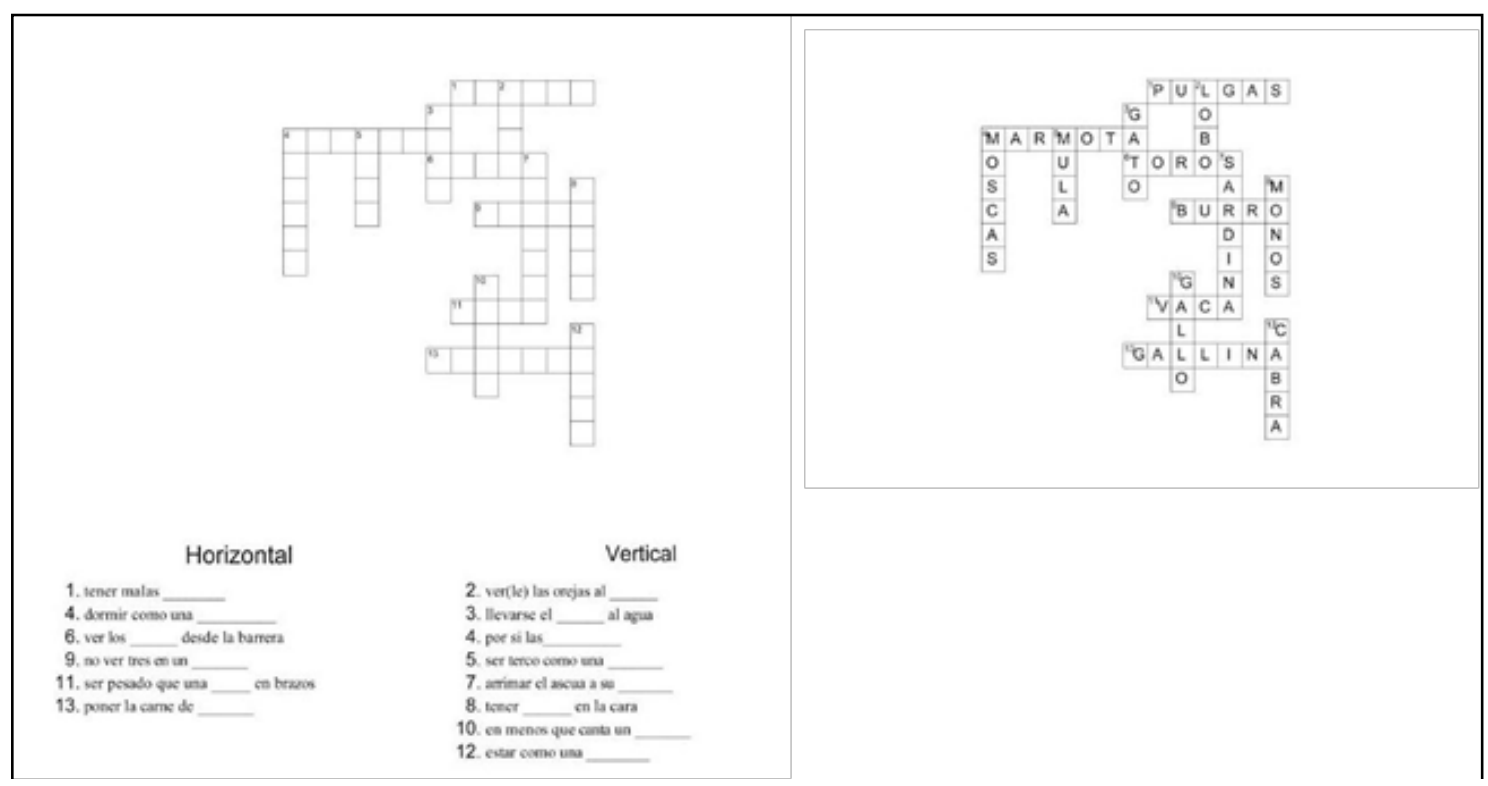

Figura 4. Capturas de pantalla de Educima, con ejemplo de creación de definiciones a través de una locución.

\subsection{Creación de crucigramas mediante el programa EclipseCrossword}

Quizá una de las opciones más completas para generar nuestros propios crucigramas y emplearse en el aula de español sea el programa gratuito EclipseCrossword ${ }^{13}$. Permite, además de titular el crucigrama que se origine, crear desde cero nuestras propias listas de palabras o modificar una lista de palabras guardada previamente, aparte de añadir alguna definición o «pista» para averiguar la palabra en cuestión (para lo que hemos empleado como ejemplo aquí, de nuevo, unidades fraseológicas con el hueco vacío de la palabra buscada). Admite la elección del tamaño del crucigrama (esto es, el número de casillas a lo largo y ancho, cosa que el programa propone automáticamente dependiendo del número de palabras que entren en juego) y lo genera automáticamente, con la opción de cambiar la visualización hasta conseguir la que creamos conveniente.

El programa también ofrece la posibilidad de imprimir en PDF el crucigrama resultante (tanto con las casillas vacías como con la solución), además de publicarlo en línea y crearlo como página interactiva de extensión .html. Cuenta, además, con una guía de uso bastante completa. Sin embargo, un inconveniente que se le puede señalar es que el idioma de ejecución está en inglés ${ }^{14}$, hecho que podría dificultar el manejo del programa al usuario cuyo dominio de dicha lengua sea limitado.

Como ejemplo de la generación de un crucigrama mediante esta útil herramienta, ofrecemos las figuras 5A-C, centrado este en el léxico de la ropa o prendas de vestir. Así, en 5A observamos dos fases distintas de uno de los pasos principales para la creación de un crucigrama con EclipseCrossword: tras seleccionar, si queremos, la

\footnotetext{
${ }^{13}$ Descargable en http://www.eclipsecrossword.com/ (fecha de consulta: 10/03/2020).

${ }^{14} \mathrm{Sin}$ ofrecer la posibilidad de cambiar de lengua, sino solamente de alfabeto.
} 
creación de una lista de palabras nuevas o la modificación de una ya existente (paso 1), mediante el paso 2 realizamos la inserción de las palabras que aparecerán en el juego. Nótese que, como «pista» para el descubrimiento de la palabra buscada, hemos planteado una locución que contiene una pieza léxica sobre la ropa como elemento integrante ${ }^{15}$, de manera que en este crucigrama el ejercicio se ha concebido como refuerzo o adquisición no solo de léxico, sino también de unidades fraseológicas en español, como el ejemplo anterior, en el apartado 2.2.

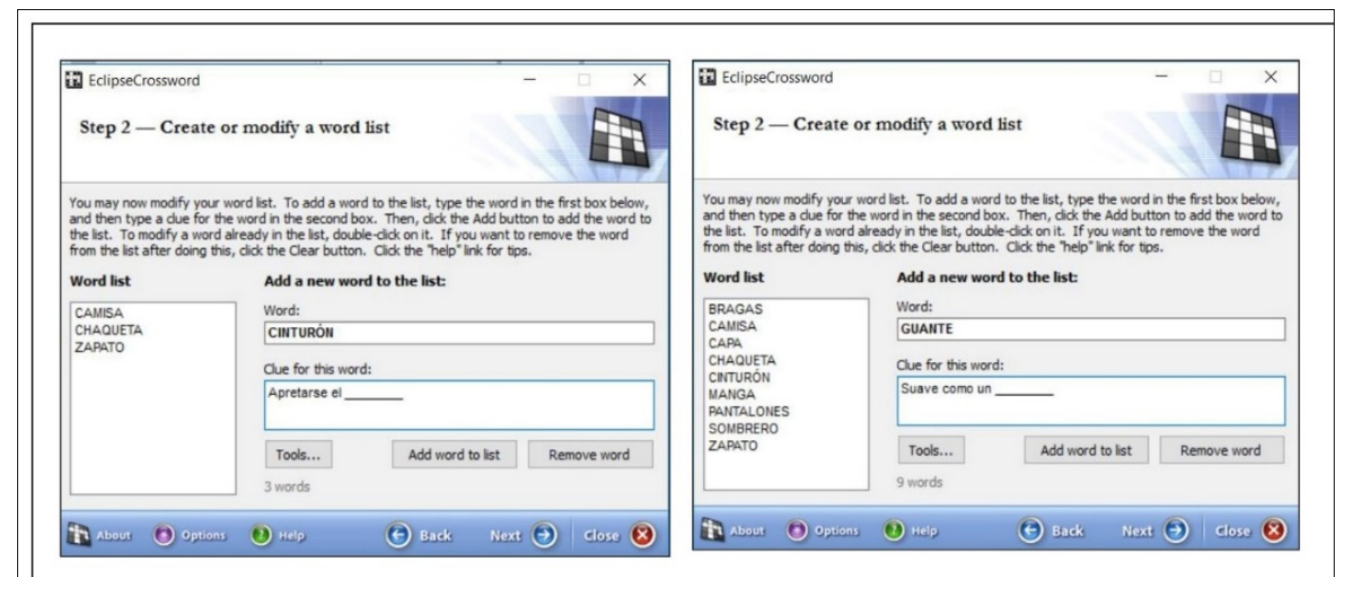

Figura 5A. Capturas de pantalla de EclipseCrossword con ejemplo de creación de definiciones a través de una locución.

Tras indicar (opcionalmente) si deseamos asignar a alguna de las palabras de nuestra lista el rol de «palabra primaria» (que aparecerá resuelta en el crucigrama y a partir de la cual pueden formarse las restantes), insertar un título para el pasatiempo y seleccionar un tamaño para el casillero -si deseamos modificar el que la herramienta nos ofrece por defecto- (pasos 3, 4 y 5), en la figura 5B podemos observar el último paso para la creación de nuestro crucigrama, en el que EclipseCrossword genera aleatoriamente una posible disposición visual con las palabras insertadas, que el programa modificará (también aleatoriamente) si así lo deseamos, hasta conseguir la presentación que creamos más apropiada para el juego.

\footnotetext{
${ }^{15}$ En concreto, sobre el léxico de la ropa, como apretarse [alguien] el cinturón ('loc. verb. Reducir los gastos por haber escasez de medios', Clave, s. v. cinturón), meterse [alguien] en camisa de once varas ('loc. verb. col. Meterse en algo que no le incumbe o que no será capaz de realizar', Clave, s. v. camisa) o cambiar [alguien] de chaqueta ('loc. verb. Cambiar interesadamente de ideas, esp. si son políticas', Clave, s. v. chaqueta).
} 


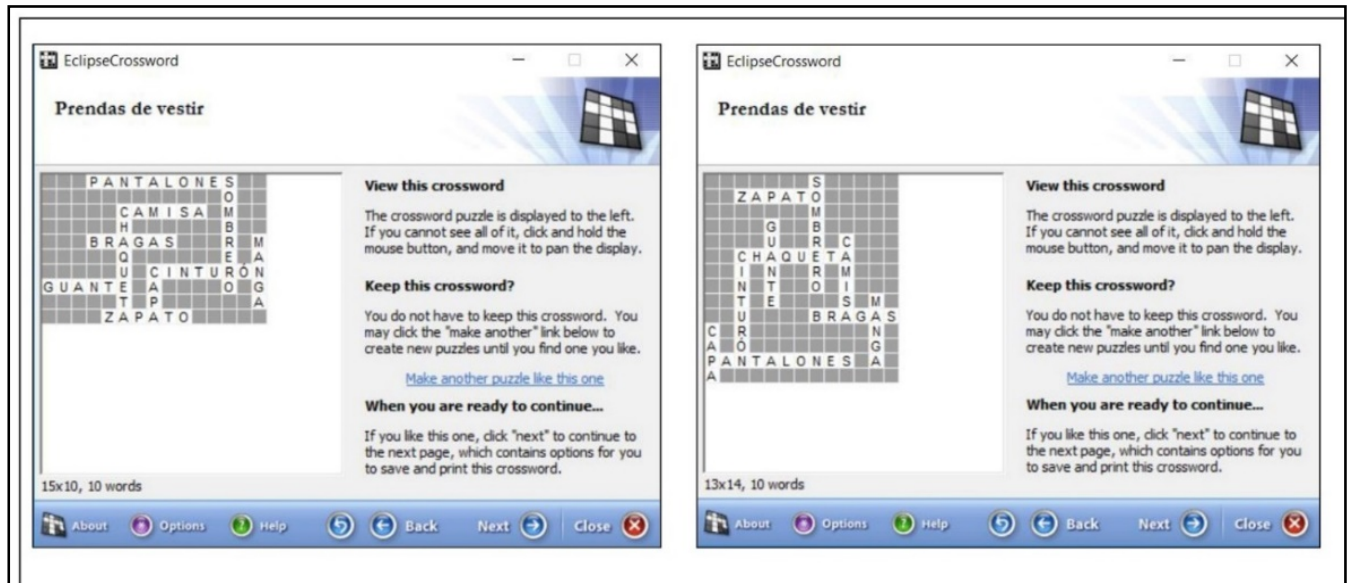

Figura 5B. Capturas de pantalla de EclipseCrossword con ejemplo de creación de dos posibles configuraciones de crucigramas.

De esta manera, podemos conseguir de forma fácil y rápida un atractivo crucigrama con EclipseCrossword sobre el campo semántico que deseemos, como podemos comprobar su finalización en la figura $5 \mathrm{C}$.

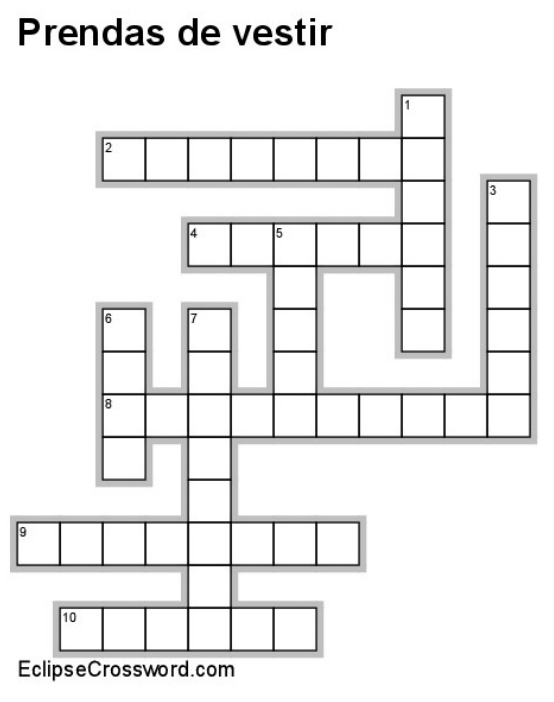

\section{Across}

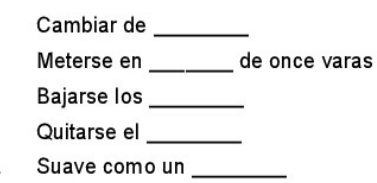

\section{Down}

1. No llegar[le a alguien] a la suela del

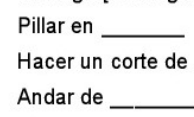

\section{SOLUCIÓN}

\section{Prendas de vestir}

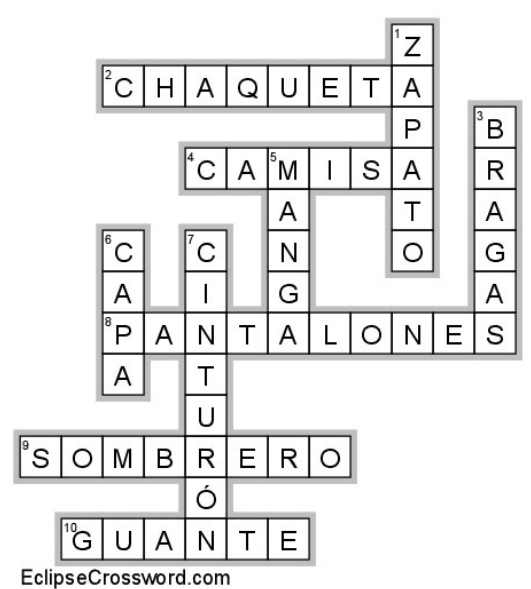

Figura 5C. Crucigrama sobre el léxico de la ropa, generado con EclipseCrossword y concebido como actividad de refuerzo/memorización de locuciones verbales usuales con una prenda de vestir como componente léxico. 
Por último, cabría destacar que esta herramienta ofrece la posibilidad de incluir en su lista de palabras piezas léxicas pluriverbales, por lo que facilitaría trabajar con unidades fraseológicas (como los distintos tipos de locuciones o, desde un punto de vista amplio, colocaciones; vid. Corpas Pastor, 1996; García-Page Sánchez, 2008) u otros tipos de construcciones conformadas con espacios en blanco en su escritura (compuestos sintagmáticos, verbos soporte, etc.). Así, por ejemplo, en la figura 6 recogemos, como actividad de refuerzo/memorización (Moreno García, 2017: 132 y ss.), un crucigrama basado en locuciones nominales relativas a características del modo de ser de una persona, tomadas del Diccionario de locuciones nominales, adjetivas y pronominales para la enseñanza del español (DICLOCNAP) de Penadés Martínez (2008).
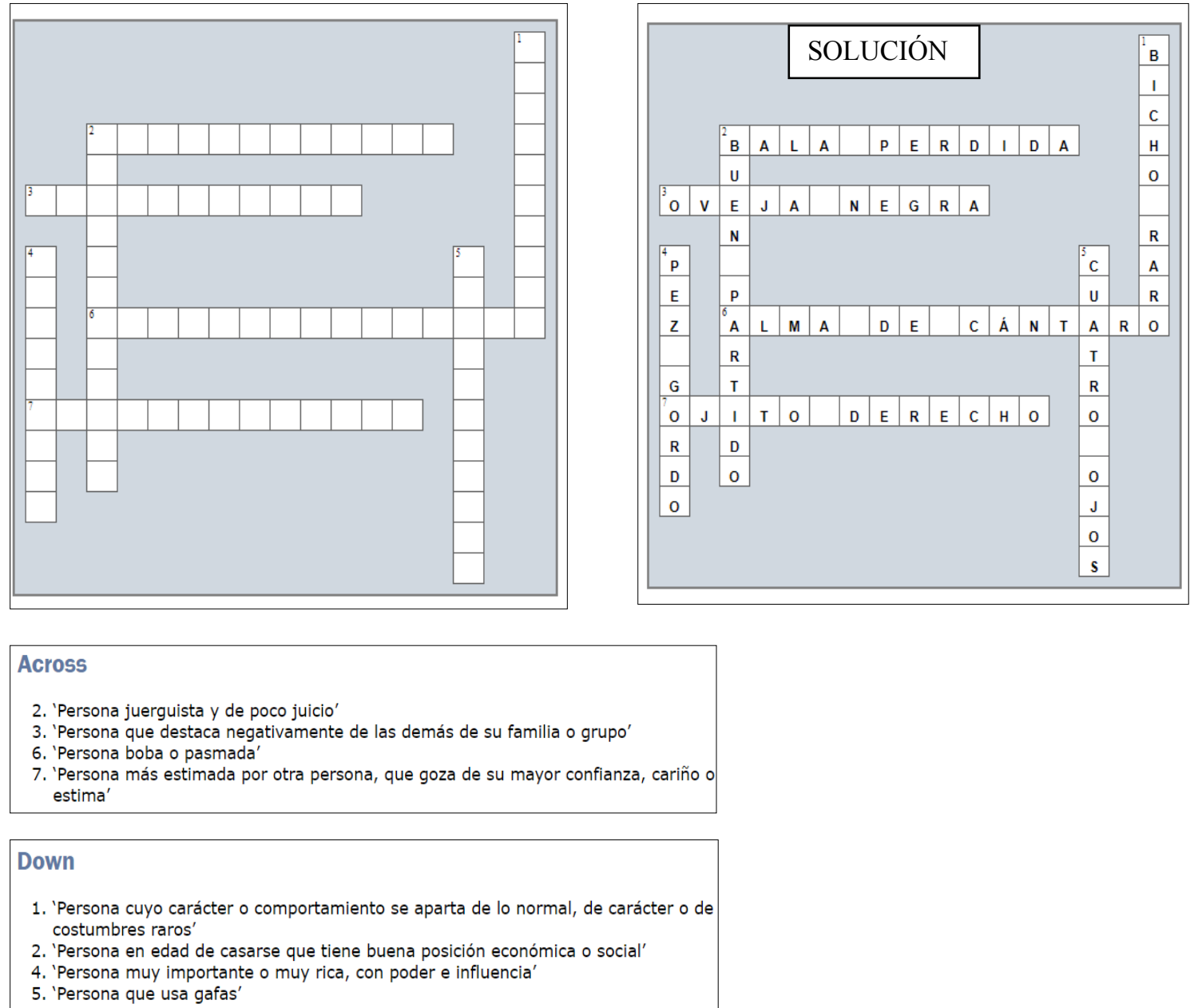

7. 'Persona más estimada por otra persona, que goza de su mayor confianza, cariño o estima'

Figura 6. Crucigrama sobre locuciones nominales que sirven para describir la personalidad, generado con EclipseCrossword y concebido como actividad de refuerzo/memorización. Las locuciones nominales integrantes se han extraido del DICLOCNAP, mientras que las definiciones empleadas como pistas se han tomado conjuntamente del DICLOCNAP y del Clave. 


\section{Conclusiones}

En el presente artículo hemos ofrecido un análisis de tres herramientas para que el docente de E/LE pueda crear y configurar crucigramas para emplearlos en el aula de español con diversos fines (adquisición-refuerzo de vocabulario de un campo léxico determinado; adquisición-refuerzo de locuciones que contienen ciertos elementos léxicos, etc.). Ateniéndonos a las posibilidades de control que cada una de estas herramientas ofrece (como el número de palabras en juego, las piezas léxicas en sí, las posibilidades de elección entre campos semánticos, el nivel de dificultad, el tamaño del juego en líneas horizontales y verticales, etc.), hemos podido comprobar que las opciones de control del juego pueden encontrarse de forma gradual: en efecto, mientras que en la primera web examinada, Crucigramas para imprimir, las posibilidades de creación y modificación de nuestros crucigramas vienen muy limitadas (lo que es representante de otros muchos generadores en línea), la opción con la que disponemos de mayor control sobre el proceso de creación del juego es en el programa descargable EclipseCrossword, ya que en él es el propio docente quien decide absolutamente las unidades léxicas concretas, su tipo de definición, el aspecto y visualización del crucigrama e incluso guardar sesiones anteriores para que se pueda reeditar.

\section{Referencias bibliográficas}

\section{Bibliografía}

Aguilar Ruiz, M. J. (2011). «Enseñanza de E/LE y fraseología: algunas consideraciones teórico-prácticas para la enseñanza de fraseología a aprendices germanohablantes (I)», Foro de Profesores de E/LE, 7. Recuperado el 10/03/2020 de: https://ojs.uv.es/index.php/foroele/article/view/6680/6469.

DOI: https://doi.org/10.7203/foroele.0.6680.

Aguilar Ruiz, M. J. (2012). «Enseñanza de E/LE y fraseología: algunas consideraciones teórico-prácticas para la enseñanza de fraseología a aprendices germanohablantes (II)», Foro de Profesores de E/LE, 8. Recuperado el 10/03/2020 de: https://ojs.uv.es/index.php/foroele/article/view/6601/6387.

DOI: https://doi.org/10.7203/foroele.0.6601.

Aguilar RUiz, M. J. (2013). «Notas sobre las posibilidades de aprendizaje de español mediante unidades fraseológicas», Marcoele. Revista de didáctica del español como lengua extranjera, 17 . Recuperado el 10/03/2020 de: https://marcoele.com/descargas/17/aguilar-unidades_fraseologicas.pdf.

Aguilar Ruiz, M. J. (2019). «Los pasatiempos como recurso didáctico en el aula de E/LE (1): Planteamientos generales. Los mensajes cifrados», Foro de Profesores de E/LE, 15, pp. 5-22. Recuperado el 09/03/2020 de: https://ojs.uv.es/index.php/foroele/article/view/14338/14501. DOI: https://doi.org/10.7203/foroele.0.14338.

Alvar EzQuerra, M. y F. Moreno Fernández (1995). DPELE. Diccionario para la enseñanza de la lengua española. Barcelona: Biblograf.

Cohen, A. y L. Frates (2010). Spanish Word Games for Dummies. Indiana: Wiley Publishing. 
Coperías Aguilar, M. ${ }^{a}$ J., J. Redondo SAnchis y J. SANMARTín SÁez (eds.) (2000). Aprendizaje y enseñanza de una segunda lengua. València: Universitat de València.

CORPAs PAstor, G. (1996). Manual de fraseología española. Madrid: Gredos.

GARCíA-PAGE SÁNCHEZ, M. (2008). Introducción a la fraseología española. Estudio de las locuciones. Barcelona: Ánthropos.

Gómez GonzÁlez, A. y C. UREÑA TORMO (2014). Locuciones y refranes para dar y tomar. El libro para aprender más de 120 locuciones y refranes del español. Niveles B2 y C1. Alcalá de Henares: Servicio de Publicaciones de la Universidad de Alcalá.

Gómez GonzÁlez, A. y C. UREÑA TORMo (2017). «Una propuesta para la enseñanza de las locuciones en la clase de E/LE», Foro de Profesores de E/LE, 13. Recuperado el 10/03/2020 de: https://ojs.uv.es/index.php/foroele/article/view/10813/10091.

Gómez MolinA, J. R. (2000): «Las unidades fraseológicas en español: una propuesta de clasificación para la enseñanza de locuciones en la clase de E/LE». En Coperías Aguilar, M. ${ }^{a}$ J., J. Redondo Sanchis y J. Sanmartín Sáez (eds.) (pp. 111-134) op. cit.

Gutiérrez CuAdrado, J. (dir.) (1996). DSLE. Diccionario Salamanca de la lengua española. Madrid: Santillana/Universidad de Salamanca.

Instituto Cervantes (1997-2019). Pasatiempos de Rayuela, del Centro Virtual Cervantes [página web]. Recuperado el 09/03/2020 de: https://cvc.cervantes.es/aula/pasatiempos/pasatiempos1/.

InSTITUTO CERVANTES (2002). MCER. Marco común europeo para las lenguas: aprendizaje, enseñanza, evaluación. Madrid: Anaya. [Traducción al español de Common European Framework of Reference for Languages: Learning, Teaching, Assessment. Strasbourg: Council of Europe, 2001]. Recuperado el 10/03/2020 de: https://cvc.cervantes.es/ensenanza/biblioteca_ele/marco/.

JACQUET, J. y S. CASUlleRAS (2006 [2004]). 40 juegos para practicar la lengua española. Barcelona: Graó.

LefEBVRE, N. (s. d.). Materiales Todoele.net: crucigramas y sopas de letras [página web]. Recuperado el 10/03/2020 de: www.todoele.net/actividades/Actividad_maint.asp?actividadespage $=3 \&$ Actividad_id $=3$ 68.

Maldonado González, M. ${ }^{a}$ C. (dir.a) (1996). Clave. Diccionario de uso del español actual. Madrid: SM.

Maldonado GonzÁlez, M. ${ }^{a}$ C. (dir. $\left.{ }^{a}\right)$ (2002). DEPE. Diccionario de español para extranjeros. Madrid: SM.

MEDINA GuerRA, A. M. ${ }^{a}$ (coord. $\left.{ }^{a}\right)$ (2003). «La microestructura del diccionario: la definición», en MEDINA GuERRA, A. M. (coord. ${ }^{\text {) }}$ (2003): Lexicografía española (pp. 127-146). Barcelona: Ariel.

MERINO GONZÁLEZ, A. (2015). «Jugando se entiende la gente. Una propuesta didáctica para la inclusión de las unidades fraseológicas en el aula de E/LE», Foro de Profesores de E/LE, 11, pp. 215-225. Recuperado el 10/03/2020 de: https://ojs.uv.es/index.php/foroele/article/view/7118/9422. 
MORENO GARCÍA, C. (2017). Materiales, estrategias y recursos para la enseñanza de español como 2/L. Madrid: Arco/Libros.

PenAdÉs MARTínez, I. (2008). DICLOCNAP. Diccionario de locuciones nominales, adjetivas y pronominales para la enseñanza del español. Madrid: Arco/Libros.

Olenych, R. (2001). Challenges Galore: Vocabulary Building Puzzles. Pensilvania: Mark Twain Media.

PORTO DAPENA, José-Álvaro (2014). La definición lexicográfica. Madrid: Arco/Libros.

PUERTAS RiBÉS, E. (2019): «Las locuciones nominales en el aula de E/LE: una propuesta para su aprendizaje», Foro de Profesores de E/LE, 15, pp. 201-221. Recuperado el 10/03/2020 de: https://ojs.uv.es/index.php/foroele/article/view/15650/14514. DOI: https://doi.org/10.7203/foroele.0.15650.

RuIZ GuRILlo, L. (2000). «Un enfoque didáctico de la fraseología española para extranjeros». En Coperías Aguilar, M. ${ }^{a}$ J., J. Redondo Sanchis y J. Sanmartín Sáez (eds.) (pp. 259-275) op. cit.

SÁNCHEZ PÉREZ, A. (coord.) (1985). GDLE. Gran diccionario de la lengua española. Madrid: SGEL.

SÁnchez Muñoz, T., J. L. Herrero Ingelmo y A. Lucas Figal (2007). DESAL. Diccionario estudio Salamanca. Barcelona: Octaedro.

Tusell, F. (1987). Diccionario del crucigramista. Barcelona: Vox/Biblograf.

VV. AA. (1974). Diccionario de crucigramas. Barcelona: Gustavo Gili.

VV. AA. (1995). Diccionario auxiliar del crucigramista. Barcelona: Vox/Biblograf.

VV. AA. (2003). Saber resolver crucigramas. Barcelona: Sopena.

\section{Webgrafía}

«Crucigramas para imprimir». Disponible en la web: http://www.crucigrama.org/index.php. Recuperado el 10/03/2020.

«EclipseCrossword». Disponible en la web: http://www.eclipsecrossword.com/. Recuperado el 10/03/2020.

«Educima». Disponible en la web: https://www.educima.com/crosswordgenerator.php. Recuperado el 10/03/2020.

«ePasatiempos». Disponible en la web: www.epasatiempos.es. Recuperado el $10 / 03 / 2020$.

«Pasatiempos de Rayuela», del Instituto Cervantes. Disponible en la web: cvc.cervantes.es/aula/pasatiempos/pasatiempos1/. Recuperado el 10/03/2020. 